\title{
TU/e EmonOWEN

\section{The initiation and progression of damage in composite overwrapped pressure vessels subjected to contact loads}

\section{Citation for published version (APA):}

Weerts, R. A. J., Cousigné, O., Kunze, K., Geers, M. G. D., \& Remmers, J. J. C. (2021). The initiation and progression of damage in composite overwrapped pressure vessels subjected to contact loads. Journal of Reinforced Plastics and Composites, 40(15-16), 594-605. https://doi.org/10.1177/0731684421992121

DOI:

10.1177/0731684421992121

Document status and date:

Published: 01/08/2021

\section{Document Version:}

Publisher's PDF, also known as Version of Record (includes final page, issue and volume numbers)

\section{Please check the document version of this publication:}

- A submitted manuscript is the version of the article upon submission and before peer-review. There can be important differences between the submitted version and the official published version of record. People interested in the research are advised to contact the author for the final version of the publication, or visit the $\mathrm{DOI}$ to the publisher's website.

- The final author version and the galley proof are versions of the publication after peer review.

- The final published version features the final layout of the paper including the volume, issue and page numbers.

Link to publication

\section{General rights}

Copyright and moral rights for the publications made accessible in the public portal are retained by the authors and/or other copyright owners and it is a condition of accessing publications that users recognise and abide by the legal requirements associated with these rights.

- Users may download and print one copy of any publication from the public portal for the purpose of private study or research.

- You may not further distribute the material or use it for any profit-making activity or commercial gain

- You may freely distribute the URL identifying the publication in the public portal.

If the publication is distributed under the terms of Article 25fa of the Dutch Copyright Act, indicated by the "Taverne" license above, please follow below link for the End User Agreement:

www.tue.nl/taverne

Take down policy

If you believe that this document breaches copyright please contact us at:

openaccess@tue.nl

providing details and we will investigate your claim. 


\title{
The initiation and progression of damage in composite overwrapped pressure vessels subjected to contact loads
}

Journal of Reinforced Plastics and Composites

202I, Vol. 40(15-16) 594-605

(C) The Author(s) 2021

Article reuse guidelines:

sagepub.com/journals-permissions DOI: $10.1177 / 0731684421992121$ journals.sagepub.com/home/jrp

(S)AGE

\author{
Ruben AJ Weerts ${ }^{12} \oplus$, Olivier Cousigné ${ }^{2}$, Klaas Kunze ${ }^{2}$, \\ Marc GD Geers' and Joris JC Remmers'
}

\begin{abstract}
In order to investigate the residual strength of composite overwrapped pressure vessels subjected to impact loads, an extensive experimental study has been performed. The vessels are loaded up to different mechanical work levels (quasistatic) or with various impact energies (dynamic). After loading, the emerged damage in the pressure vessel is examined using computed tomography scans. The tests of vessels up to various energies enable us to visualize the initiation and progressive growth of damage. The overall influence of the damage is subsequently quantified by means of a burst pressure test. Accordingly, the reduction of burst pressure can be used to evaluate the global severity of the observed damage in the computed tomography scan. From the experiments, it is concluded that the first failure mechanisms, that is, delaminations, do not cause a meaningful reduction of the burst pressure. Shear bands, on the other hand, which are formed thereafter, do induce a significant decrease of the residual strength.
\end{abstract}

\section{Keywords \\ Damage tolerance, impact behavior, computed tomography analysis, mechanical testing}

\section{Introduction}

Recent studies show the potential of hydrogen technology in terms of decarbonizing our future energy supply. Staffell et al. ${ }^{1}$ concluded that technologies such as hydrogen boilers, fuel cell CHPs, and hydrogen powered heat pumps could support future heat generation. They stated that only small adaptations to the existing natural gas network are required. Hydrogen could also be used to store excess electrical energy. As the energy delivered from wind and solar energy is not constant over time, energy needs to be stored to balance the energy supply. It is possible to convert this electrical energy in hydrogen to store it. ${ }^{2,3}$ The stored hydrogen can be transformed back into electrical energy when required. Alternatively, it could also be used to power fuel cell electric vehicles (FCEVs). In these vehicles, stored hydrogen is converted into electricity for their propulsion. Studies show that the total greenhouse gas emissions are in many cases lower for these FCEVs than for diesel or even battery electric vehicles (BEVs) when the complete lifetime of the vehicle is considered. ${ }^{4,5}$ Moreover, the required investment to build the infrastructure is comparable or even less for FCEVs than for BEVs. ${ }^{6}$

As the volumetric energy density of hydrogen at ambient conditions is low, efficient storage of hydrogen is a key topic. Barthelemy et al. ${ }^{7}$ compared various technologies to store hydrogen, concluding that cryogenic and compressed storage are the most mature technologies. Cryogenic storage is a method in which liquid hydrogen is stored in an insulated vessel at approximately $-250^{\circ} \mathrm{C}$. This technique is often used for delivery of hydrogen, but due to loss by evaporation, it is unsuitable where longtime storage is required, as in vehicles. ${ }^{7}$ Instead, for FCEVs, it is more common to store the hydrogen under high pressure (up to 700 bar) in, for example, composite overwrapped pressure vessels (COPVs). The composite overwrap is used to withstand the internal pressure. As it can be subjected to various load cases that may cause barely visible damage, for example, falling during handling, the influence of the initiated complex damage on the residual lifetime needs to be well understood. ${ }^{7,8}$

Different inspection methods have been proposed to assess the condition of a pressure vessel while it is still in

\footnotetext{
'Mechanics of Materials, Department of Mechanical Engineering, Eindhoven University of Technology, The Netherlands

${ }^{2}$ Department of Hydrogen Supply, BMW AG, Germany
}

\section{Corresponding author:}

Ruben AJ Weerts, Department of Hydrogen Supply, BMW AG, Petuelring 130, Munich 80788, Germany.

Email: Ruben.Weerts@bmw.de 
service. Choi et al. ${ }^{9}$ used aluminum-coating optical fibers to detect impacts on composite pressure vessels. In this study, a fiber was placed along the surface of a vessel, and the pressure vessel was subjected to multiple impacts. The impact locations could be exactly determined using the measured Brillouin frequency along the fiber. Small impact traces could be detected, which did not cause critical structural degradation. ${ }^{9}$ Saeter et al. ${ }^{10}$ proposed an inspection method in which optical fibers are embedded in the composite overwrap as a network of distributed strain sensors. The fibers were used to measure strains in several thicknesses of the composite layup and could also be used to detect damage after impact by analyzing the backscattered light. Liao et al. ${ }^{11}$ used acoustic emission to recognize failure during hydraulic testing. A relation was found between the emitted sound and the corresponding failure event. Using this result, the initiation and growth of failure close to bursting was studied. Such techniques could be used in periodical inspections of COPVs to identify unnoticed damages.

A crash poses an additional safety concern as it leads to a sudden reduction of residual strength. Blanc-Vannet et al. ${ }^{12}$ conducted impacts on the cylindrical area of pressure vessels. Burst tests were conducted after impacting a vessel to establish a relationship between the incident energy and the residual burst pressure. In a different study, BlancVannet ${ }^{13}$ summarized a large series of impacts on 200 pressure vessels with a wide range of thicknesses (5$30 \mathrm{~mm})$ and impact energies $(6-11,000 \mathrm{~J})$. Furthermore, a comparison of the effect of impact on unpressurized and pressurized pressure vessels was made. For unpressurized cylinders, a global deformation pattern which remains permanent after unloading was found. On the other hand, for pressurized cylinders, damage is located right under the impact location. It was concluded that empty cylinders absorb a larger fraction of incident energy than cylinders with an internal pressure of 700 bar $(80 \%$ versus $60 \%)$. For both empty and pressurized cylinders, the burst pressure starts to decrease after passing a certain threshold impact energy. Blanc-Vannet ${ }^{13}$ found a relationship to determine this threshold energy for pressure vessels with different initial burst pressures and different inner radii.

Garcia et al. ${ }^{14}$ tested both unpressurized and pressurized $(<300$ bar) vessels under hypervelocity impact conditions. Various projectile masses, velocities, and diameters were used. In addition, different impact locations and angles were considered. The damage ranged from penetration of the carbon fiber reinforced polymer (CFRP) without perforating the metallic liner up to full perforation of the wall (CFRP and liner). The COPVs that passed testing (i.e., no burst) did so while incurring full perforation of the $2.5-\mathrm{mm}$ thick CFRP overwrap. ${ }^{14}$ Demir et al. ${ }^{15}$ studied the effects of repeated impacts and of different temperatures during impact on the residual burst pressure of pressure vessels after the impact. The considered vessels had a CFRP thickness of
$4 \mathrm{~mm}$, and impact energies were ranging from 10 to $30 \mathrm{~J}$. It was shown that the residual burst pressure of vessels exposed to repetitive impact loads is lower than pressure vessels which were only exposed to single impact loads. To study the effect of temperature, impact tests were conducted on vessels that were filled with water at a temperature of either 25 or $70^{\circ} \mathrm{C}$. A subsequent burst pressure test showed that impacts at an increased temperature resulted in a larger reduction of the residual burst pressure. Perillo et al. ${ }^{16}$ conducted impacts on glass fiber reinforced pressure vessels and considered impacts of small impactors with a diameter of $16 \mathrm{~mm}$. The induced energy ranged from 20 up to $100 \mathrm{~J}$. The observed failure mechanisms consisted of matrix cracking, delaminations, and fiber failure. ${ }^{16}$

Although various studies exist reporting impacts on COPVs, the relation between external loading, the initiation and growth of failure, and their influence on the residual strength of the vessel has not been identified. In order to investigate this relation, the present study considers various unpressurized COPVs that are subjected to quasi-static and dynamic contact loads. The vessels are loaded up to various deformations or using different impact energies in order to study the progression of damage. By investigating both quasi-static and dynamic cases, the role of inertia effects and stress waves can be identified. In addition, the combined quasi-static and dynamic response information is useful for the future development of numerical models analyzing the impact behavior. Finally, the vessels are examined by means of computed tomography (CT) images to identify the location and amount of induced damage. After this investigation, the vessels are subjected to a burst test in order to determine their residual burst strength. The reduction in burst pressure is correlated to the original impact energies and the observed damage in the $\mathrm{CT}$ images.

\section{Experimental methodology}

Closed, unpressurized pressure vessels are tested under either quasi-static or dynamic (impact) contact loads. After the contact load, the damage in the pressure vessel is assessed using a CT scan. The structural influence of the observed damage is then quantified using a burst pressure test. In this section, the analyzed pressure vessels and the applied methodology for each of these steps are presented.

\section{Tested pressure vessel}

A commercial type IV pressure vessel with typical automotive dimensions and specifications is considered. The vessel with an inner volume of $36 \mathrm{~L}$ was manufactured by Hexagon Purus and is used in the literature for impact tests more often, see for example. ${ }^{12}$ The nominal working pressure of the engineering vessel is according to state-ofthe-art technology (700 bar). The burst pressure of an undamaged vessel is at least 1575 bar (taken into account 
a safety factor of 2.25), which is a requirement of regulation (EC) No. 79/2009. The length, diameter, and wall thickness are approximately $1000 \mathrm{~mm}, 350 \mathrm{~mm}$, and $25 \mathrm{~mm}$, respectively. The vessel consists of approximately 100 plies, each with a thickness of $0.25 \mathrm{~mm}$. These plies compose a multidirectional layup, which consists of orientations between $20^{\circ}$ and $90^{\circ}$, with $0^{\circ}$ being the axial direction of the pressure vessel. The composite material consists of carbon fibers embedded in an epoxy resin matrix. The pressure vessel has similar material properties as the one presented in. ${ }^{17}$

\section{Experimental setup}

The quasi-static and dynamic contact tests were conducted at the Institute of Lightweight Engineering and Polymer Technology, Technical University of Dresden. The cylindrical section of a closed, unpressurized tank was loaded by an indenter up to a defined displacement (quasi-static) or with a specific impact energy (dynamic). A cubic shaped indenter with contact area dimensions of $100 \times 100 \mathrm{~mm}$ was used as it represents the typical shape and size of neighboring components that may impact a pressure vessel during a crash. The contact area edges were rounded with a radius of $5 \mathrm{~mm}$ to prevent local cutting of the COPV. The pressure vessels were mounted on support blocks to prevent the COPV from moving during loading. In all conducted tests, the loading caused damage initiating at the indenter/ impactor location.

More specifically, the quasi-static tests were executed using a servo-hydraulic universal testing machine. Figure 1 shows an illustration of the experimental setup. The indenter was attached to a base plate, which was placed on a load cell. The load cell measured the force that was exerted on the indenter and hence the pressure vessel. The COPV, mounted above the indenter, was connected to the top plate through the V-shaped support blocks. Initially, the COPV was kept in place using span tapes, which were attached to the top plate (not shown in the figure). As these span tapes were only loosely secured, their influence is expected to be small. The quasi-static loading was applied by moving the load cell upward at a slow rate of less than $5 \mathrm{~mm} / \mathrm{min}$. The displacement was measured through the machine crosshead displacement. Inaccuracies due to machine stiffness are expected to be small, due to the high axial stiffness of the 4-column construction of the machine.

A drop tower was used to conduct the dynamic impact tests on unpressurized pressure vessels. This setup is shown in Figure 2. The indenter was attached to a carriage which was guided by two or four columns, depending on the impact mass. If required, the impact mass of the sled was increased by adding weights, which were attached to the carriage. The impact velocity was between 3 and $8 \mathrm{~m} / \mathrm{s}$ for the conducted tests. A system was in place to catch the carriage upon rebound, preventing it from impacting the

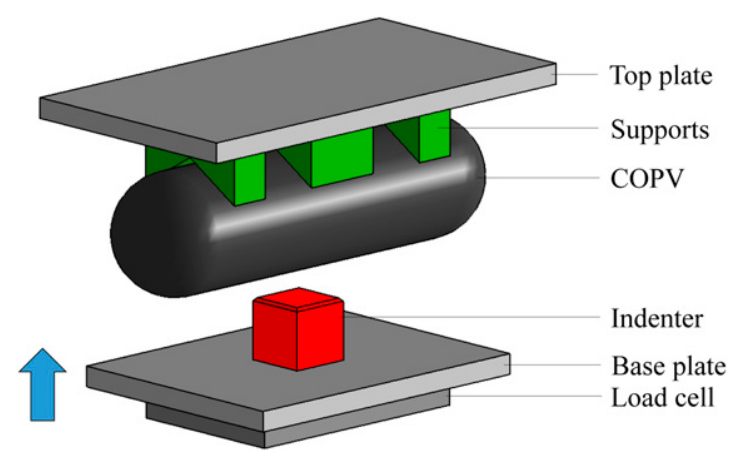

Figure I. Experimental setup for quasi-static contact loading. The indenter and base plate move toward the specimen. The used span tapes to hold the composite overwrapped pressure vessels to the top plate are not depicted.

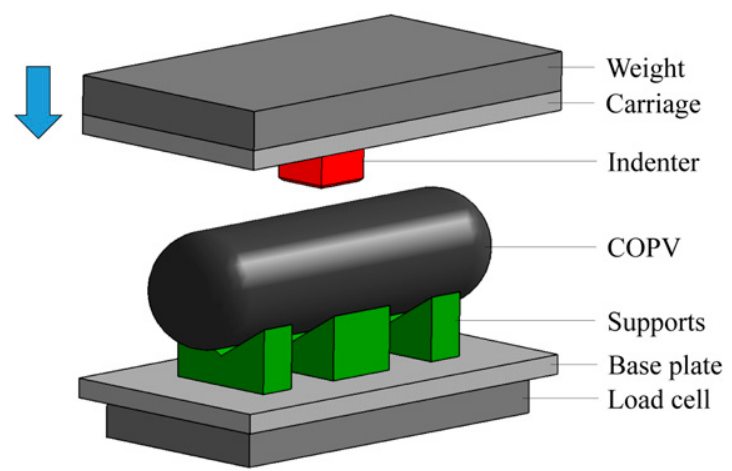

Figure 2. Experimental setup for dynamic contact loading. The used span tapes to hold the composite overwrapped pressure vessels on the base plate are not depicted.

COPV multiple times. The COPV was mounted underneath the indenter, resting on the V-shaped supports which were placed on the base plate. Span tapes were used to avoid bouncing of the COPV (not depicted in illustration). Twelve load cells were positioned underneath the base plate to measure the transmitted force during the test. This setup of multiple load cells was used to avoid too high a compliance of the force measurement device. The tests were recorded using a high-speed camera to determine the impactor position using digital image correlation. The high frame rate of over $5 \mathrm{kHz}$ required a high light intensity, which was achieved using multiple high-power lights. To prevent reflection off the CFRP layers, a matt coating was applied on the surface of the pressure vessel.

\section{CT images}

Each of the pressure vessels was studied using CT after being impacted. These scans were performed at the Institute of Lightweight Engineering and Polymer Technology, Technical University of Dresden. The used setup 
can accommodate the entire pressure vessel without having to section it in smaller samples. The latter is a limitation for most CT scanners but was not acceptable for this project as it would disable a subsequent burst pressure test. The impact location was marked before scanning, since identification of the location is difficult if only minor damage is inflicted. First, a coarse scan was conducted in order to determine the size of the damaged region. The area and resolution of the subsequent detailed scan were set to limit the scan time to approximately $4 \mathrm{~h}$. The resolution ranged between 80 and $160 \mu \mathrm{m}$, which is smaller than the ply thickness, which is about $250 \mu \mathrm{m}$.

\section{Burst tests}

To quantify the reduction of the strength due to the imposed damage, each pressure vessel was subjected to a so-called burst test. These burst tests were performed hydraulically using a pressure ramp of $3 \mathrm{bar} / \mathrm{min}$ up to $20 \mathrm{bar}$ and $120 \mathrm{bar} /$ min thereafter up to burst, that is, the moment at which the vessel wall can no longer withstand the internal pressure.

\section{Results and discussion}

The quasi-static contact tests are discussed as a benchmark first. The acquired force, displacement, and energy are normalized throughout this section for confidentiality reasons. The normalization is uniform: the largest force, displacement, and energy of the vessel with the largest deformation (QS-5) are set to 1.0, and the results for the other samples are scaled accordingly, see Table 1. The dynamic contact tests are discussed afterward for comparison with the quasi-static loading. The time of impact of vessel Dyn-3(I) is used for the normalization of time. The occurring damage is subsequently studied using the CT scans, followed by the burst tests.

Table I. Normalization of data.

\begin{tabular}{ll}
\hline Physical quantity & Normalized by \\
\hline Displacement & QS-5 \\
Force & QS-5 \\
Energy & QS-5 \\
Time & Dyn-3(I) \\
\hline
\end{tabular}

\section{Quasi-static tests}

The quasi-static tests are performed using the experimental setup previously presented. Five pressure vessels are considered, which are tested at different maximum displacements, see Table 2. The maximum applied displacement of vessel QS-5 is used to normalize all other displacements.

Figure 3 shows the measured force-displacement curve for pressure vessel QS-1, which is subjected to the smallest displacement. Initial loading is almost linear up to the first significant nonlinearity, which is referred to as first failure and indicated by point $A$. Subsequent loading continues at an almost constant force up to B, after which the sample was unloaded. Initially, the unloading stiffness is close to the initial loading stiffness. At a normalized force of approximately 0.25 , the stiffness considerably reduces up to the unloaded state (C). Some permanent deformation can be recognized after unloading. Note that the loads are normalized by the maximum reaction force measured for vessel QS-5.

The mechanical work as a function of displacement is calculated by piecewise integration of the forcedisplacement curve and shown in Figure 4. The three points introduced in the force-displacement curve are indicated in this diagram. The work at $\mathrm{A}$ is the amount of energy that can be absorbed before first failure occurs. The maximum mechanical work is shown at $\mathrm{B}$. Point $\mathrm{C}$ reflects the amount of dissipated energy, which is approximately $55 \%$ of the maximum mechanical work. Hence, approximately $45 \%$ of the mechanical work was stored elastically. In the case of a crash, we are mainly interested in the amount of incident energy that a pressure vessel can withstand instead of the energy that can be dissipated. Therefore, the remainder of this study focuses on the maximum work.

The force-displacement curves for the five tested pressure vessels (QS1-QS5) are shown in Figure 5. The unloading path of the curves is not shown for clarity purposes but follows similar trends as the unloading path of QS-1 in Figure 3. Comparison of the different curves demonstrates that the quasi-static test is highly reproducible. After the plateau, the force increases at a consistent slope for all samples. Sample QS-5 shows two force drops at deformation levels that exceed those used in the other samples.

To facilitate the comparison of the curves, the key characteristics are summarized in Table 2. The maximum

Table 2. Overview of the quasi-static tests.

\begin{tabular}{llll}
\hline Sample & Applied norm. displacement [-] & Max. norm. mechanical work [-] & Norm. force at first failure [-] \\
\hline QS-I & 0.06 & 0.024 & 0.41 \\
QS-2 & 0.12 & 0.063 & 0.46 \\
QS-3 & 0.2 & 0.13 & 0.50 \\
QS-4 & 0.27 & 0.18 & 0.48 \\
QS-5 & 1.0 & 1.0 & 0.52 \\
\hline
\end{tabular}




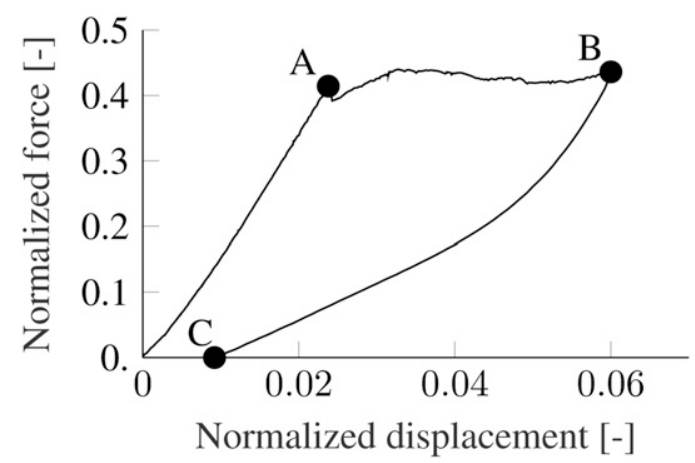

Figure 3. Normalized force-displacement curve for sample QS-I.

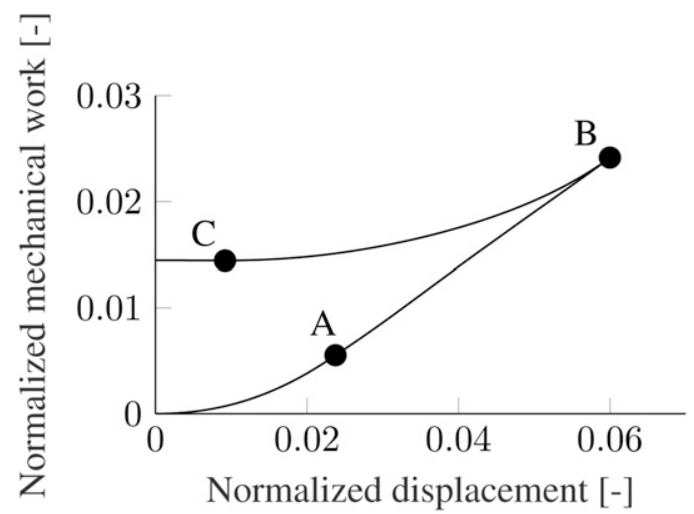

Figure 4. Normalized mechanical work-displacement curve for sample QS-I.

work directly scales with the applied displacement, although the relationship is not linear. Since the force increases as the displacement does, additional displacement leads to increasingly more energy absorption per unit of displacement. For the force at first failure, a spread of approximately $20 \%$ can be recognized between the minimum and maximum value.

\section{Dynamic tests}

The dynamic tests are performed at six incident energy levels, referred to as Dyn-1 to Dyn-6, see Table 3.

To validate the repeatability of the testing procedure, energy level Dyn-3 is tested three times: Dyn-3(I), Dyn3(II), and Dyn-3(III). Figure 6 shows the measured forcetime curves, which are clearly oscillating for each of the three samples. Yet, within the limits of what can be achieved, the tests are sufficiently reproducible as the main characteristics match; for example, the peak forces and the frequency of the oscillations. The time is normalized using the duration of the impact from sample Dyn-3(I), as can be read from Table 1.

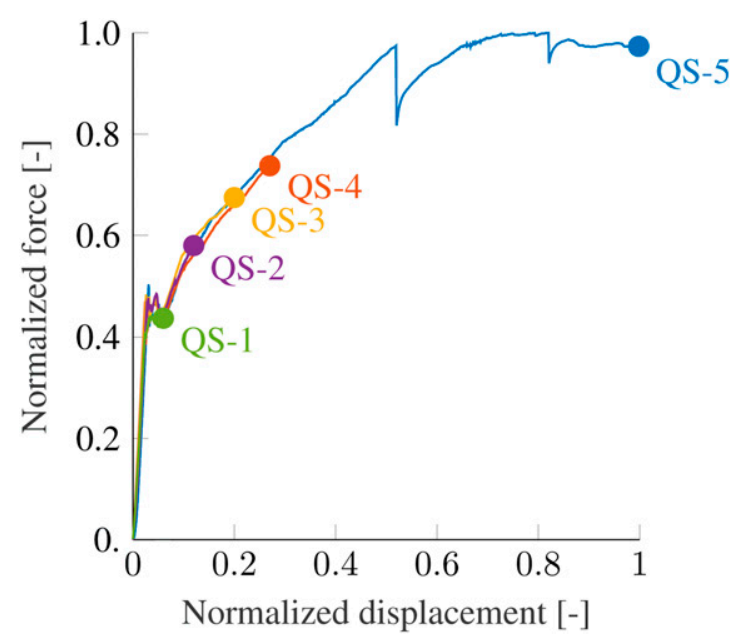

Figure 5. Normalized force-displacement curves for samples QS-I to QS-5.

The conversion of the force-time curves into forcedisplacement curves requires the combination of measurements from the load cells and the camera to obtain the force and displacement, which are shown in Figure 7. The same normalization is used as in the quasi-static study. Thus, the maximum force and displacement for vessel QS-5 are set to 1.0, and the dynamic results are scaled accordingly, see Table 1. Zero displacement is defined as the time at which the velocity of the impactor is first considerably reduced. ${ }^{1}$ The unloading paths of the curves are not depicted to improve clarity. The measured force does not increase until a normalized displacement of about 0.03 is reached. There is a time delay between the moment of impact and the moment at which the load cell positioned below the COPV registers a force.

For comparison, the quasi-static curve of the sample QS5 is included. Clearly, the dynamic curves oscillate around a trend line which corresponds with the quasi-static measurement. The first peak force in the dynamic curves is approximately twice the force at the first significant reduction of stiffness in the quasi-static case. This is caused by the "dynamic amplification factor"; for a simple vibrating system, the sudden application of loading on a mass causes oscillations reaching amplitudes that are twice as high compared to a quasi-static load, as demonstrated in. ${ }^{18}$

The occurrence of this effect for the used experimental setup is analyzed in more detail in the Appendix. The analysis reveals that wave reflections make the force measurement unfit for general conclusions. Therefore, the subsequent analysis does not exploit the force measurement further but focuses on the energy instead.

For the dynamic tests, it is assumed that most of the impact energy is converted into mechanical work in the pressure vessel. Obviously, a small part of the energy is transformed into vibrational energy or dissipated in the 
Table 3. Overview of the dynamic tests. Note that some of the dynamic samples have equivalent quasi-static samples in terms of incident energies and displacements, see also Table 2.

\begin{tabular}{lccc}
\hline Sample & Norm. incident energy [-] & Max. norm. displacement [-] & Equivalent QS sample \\
\hline Dyn-I & 0.022 & 0.045 & QS-I \\
Dyn-2 & 0.062 & 0.11 & QS-2 \\
Dyn-3(I) & 0.090 & 0.16 & 0.16 \\
Dyn-3(II) & 0.087 & 0.15 & \\
Dyn-3(III) & 0.087 & 0.21 & QS-3 \\
Dyn-4 & 0.13 & 0.27 & QS-4 \\
Dyn-5 & 0.18 & 0.62 & \\
Dyn-6 & 0.52 & & \\
\hline
\end{tabular}

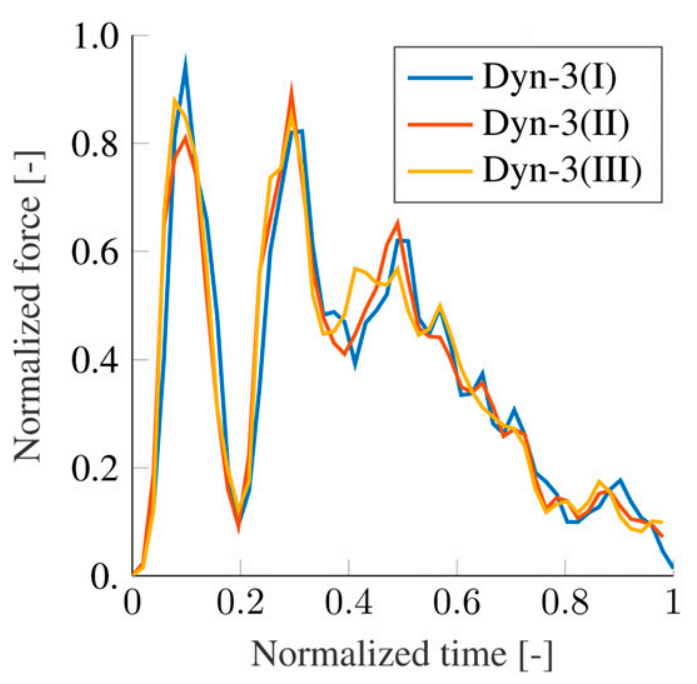

Figure 6. Normalized force-time curves for samples Dyn-3(I), Dyn-3(II), and Dyn-3(III).

experimental setup. A few of the dynamic samples were tested with an incident energy that matches the maximum applied mechanical work of a quasi-static sample, see Table 3. Comparison of the maximum displacement of those equivalent samples shows that the dynamic maximum displacement is slightly lower than its quasi-static counterpart for small incident energies but is almost equal to it for larger energies. Hence, it can be concluded that the dynamic losses are comparably small when the incident energy is sufficiently large.

Although small, the exact amount of energy that is not converted into mechanical work cannot be determined. Therefore, we will only consider the external work, which is equal to the reduction of the kinetic energy of the indenter during the impact. As a result of this definition, the external work includes the mechanical work but also vibrational energy and energy that is dissipated in the experimental setup. Figure 8 shows the normalized external work as a function of the displacement for the three dynamic samples Dyn-3(I) to Dyn-3(III) and for the quasi-static sample QS-5. The external work for the quasi-static case

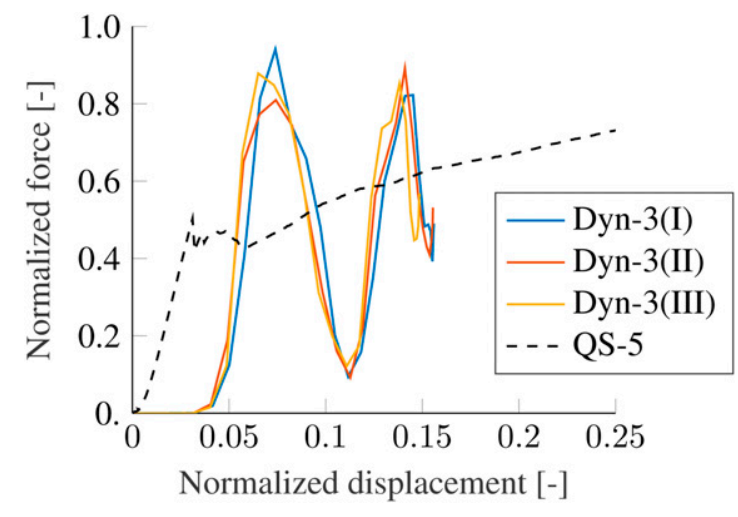

Figure 7. Normalized force-displacement curves for samples Dyn-3(I), Dyn-3(II), and Dyn-3(III). Sample QS-5 is included for reference.

is equal to the previously calculated mechanical work. The results are normalized by the same factors as for the quasistatic tests and the maximum mechanical work for sample QS-5.

At small displacements, the external work is increasing more steeply for the dynamic tests than for the quasi-static case. Due to inertia effects in the dynamic case, loading first induces deformations in the upper part of the COPV and spreads thereafter. As loading progresses and the velocity of the impactor decreases, the loading state in the COPV can reach a near quasi-static state. Therefore, at maximum work, the quasi-static case and the dynamic cases closely match. Upon unloading, part of the stored energy is converted back into kinetic energy of the impactor. Approximately $75 \%$ of the incident energy is dissipated, closely matching earlier observations by Blanc-Vannet. ${ }^{13}$

An overview of the results for all dynamic tests is shown in Figure 9. The dynamic tests show a behavior that is consistent with the quasi-static case. However, for low energies (normalized work $<0.2$ ), the corresponding displacement is smaller for the impact tests than the quasi-static ones. This suggests that the time of contact may be insufficient for the loading to reach a near quasi-static state. Alternatively, it could indicate that mechanisms such as 


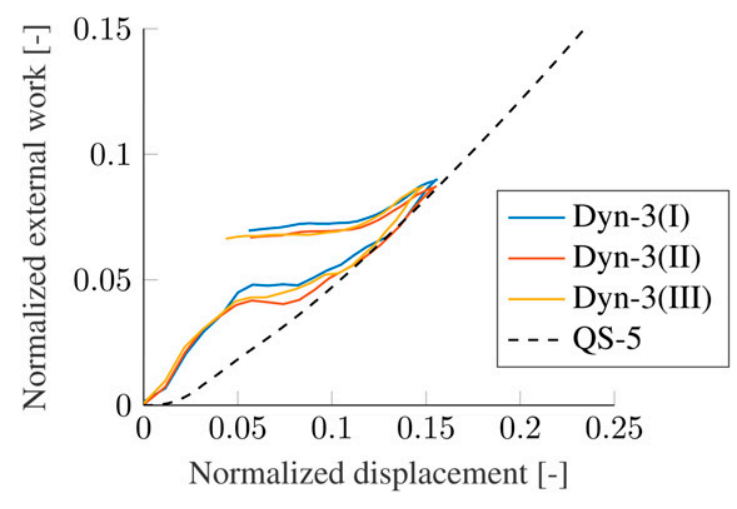

Figure 8. Normalized external work-displacement curves for samples Dyn-1, Dyn-2, and Dyn-3. Sample QS-5 is included for reference.

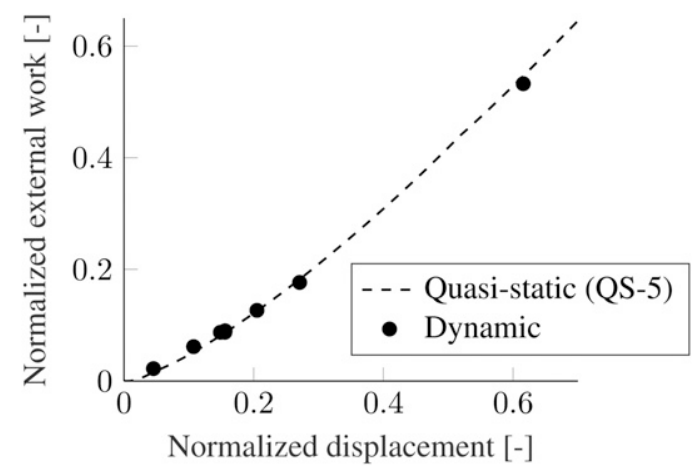

Figure 9. Normalized external work-displacement diagram for the dynamic measurements. The maximum external work is indicated using filled circles, and the quasi-static curve of QS-5 is included for reference.

friction are more significant when the incident energy is small. For large impact energies, the final displacement of the dynamic case slightly exceeds the quasi-static value.

\section{CT images}

The imposed damage is studied by means of CT images that are made from the pressure vessels after the quasi-static test but before the burst pressure tests. With a complete vessel in the CT scan, the resolution is relatively coarse but still allows for identification of individual layers and damage. In the analysis, an area of the circumferential cross section directly underneath the impactor is considered. The cross section is examined at different longitudinal positions with respect to the impactor, indicated as locations 1-9. These locations are shown in Figure 10: Position 5 is at the center underneath the impactor, and positions 2 and 8 are exactly under the edges of the impactor. Positions 1 and 9 are outside the indenter impact area.

Let us first consider pressure vessel QS-3 to identify typical damage mechanisms. Figure 11 shows the circumferential

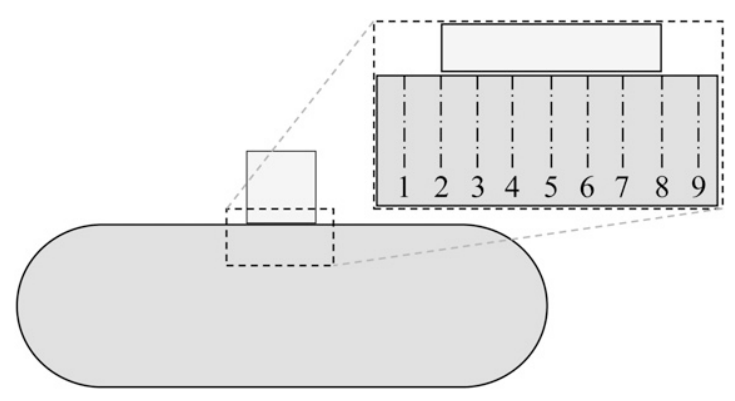

Figure 10. Longitudinal locations for the CT images. The cross sections for the CT study are taken at these locations. CT: computed tomography.

cross section at the longitudinal locations. One of the main observed failure mechanisms is delamination. This can be recognized from the cracks that develop in circumferential direction, as indicated for location 3, for example. A second failure mechanism is shear band formation, which is visible at the left side of locations 2-8 (indicated at location 5). The observed shear band consists of matrix cracking and fiber kinking, both initiating under shear-dominated loading. Either or both mechanisms can be present in a single ply, depending on its orientation. It is known that fiber kinking does not always and not immediately involve fiber fracture. ${ }^{19,20}$ For the shown shear band, fiber fracture did locally occur, which can be concluded as some (near) circumferential layers are locally split in the shown image. The shear band progresses at an angle close to $45^{\circ}$ with respect to the local radial direction and is oriented toward the contact line with the impactor. The shear band does not progress far outside the contact region as it is hardly visible at locations 1 and 9. At the right side, a less pronounced shear band emerges (see location 6). Although less visible, the right shear band also extends over the entire impactor length (28). Note that even though the test itself is symmetric, damage is obviously not.

Figure 12 shows CT images taken from the pressure vessels QS-1 to QS-5 after the quasi-static tests. The images shown are taken at the edge of the impactor, that is, location 2 in Figure 10. As the samples are tested up to increasing deformations, the images from the different pressure vessels reflect the growth of damage at increasing deformation levels. The image from pressure vessel QS-1 reveals that the first observed failure mechanism consists of delaminations. Hence, the first kink in the force-displacement curve (point A in Figure 3) is thought to be caused by the initiation of delamination. This theory is supported by earlier observations in, ${ }^{21}$ in which thick-walled short composite cylinders were loaded in a similar manner as the pressure vessels here. It was shown that the first force drop in the force-displacement curve for the samples with a wall thickness close to that of pressure vessels was accompanied with a visible growth of delamination. Note that locally, 

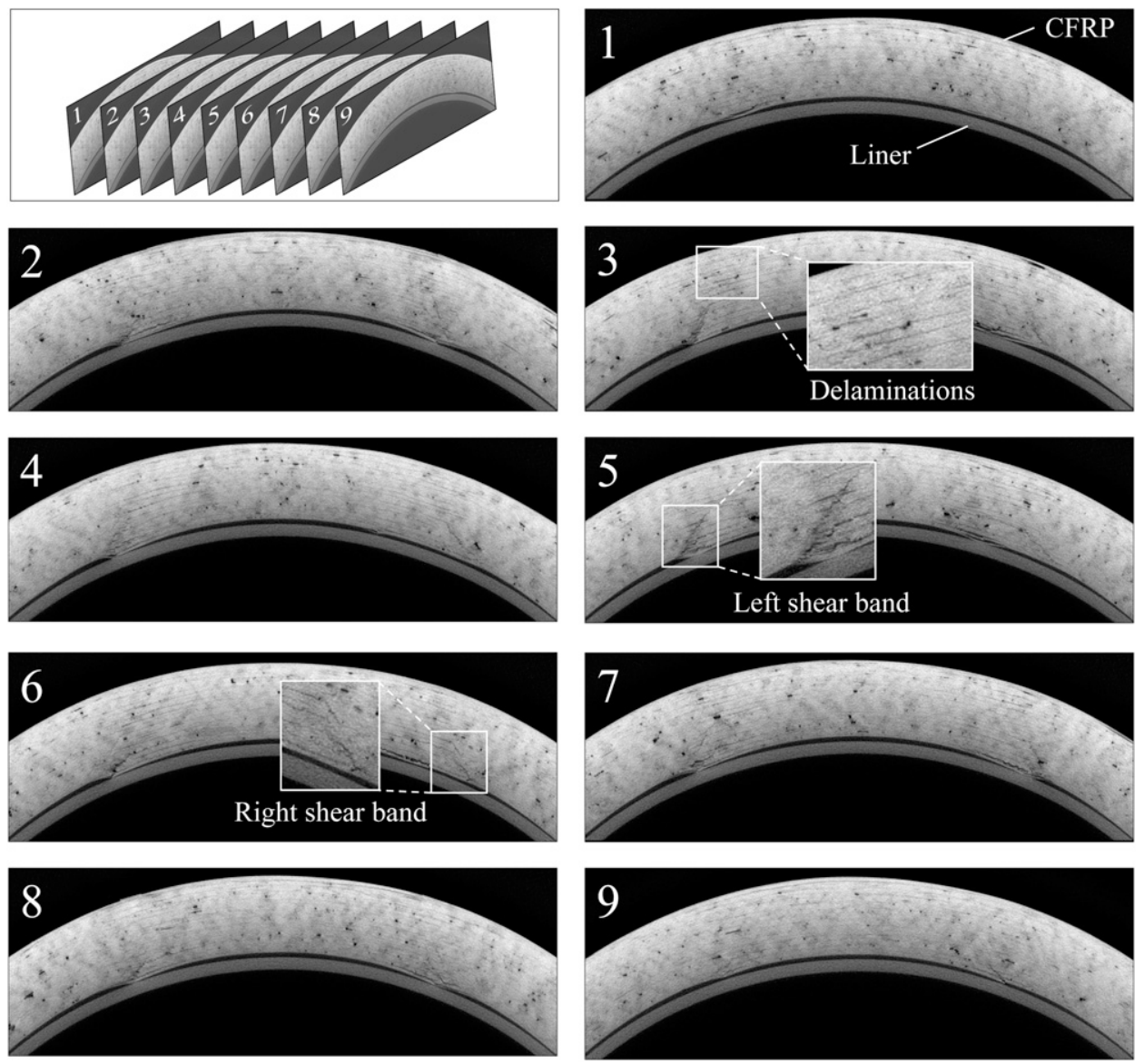

Figure I I. Computed tomography images of pressure vessel QS-3 at the locations defined in Figure 10. The most important components (liner and carbon fiber reinforced polymer) and the occurring failure mechanisms are indicated. Air is shown in black.
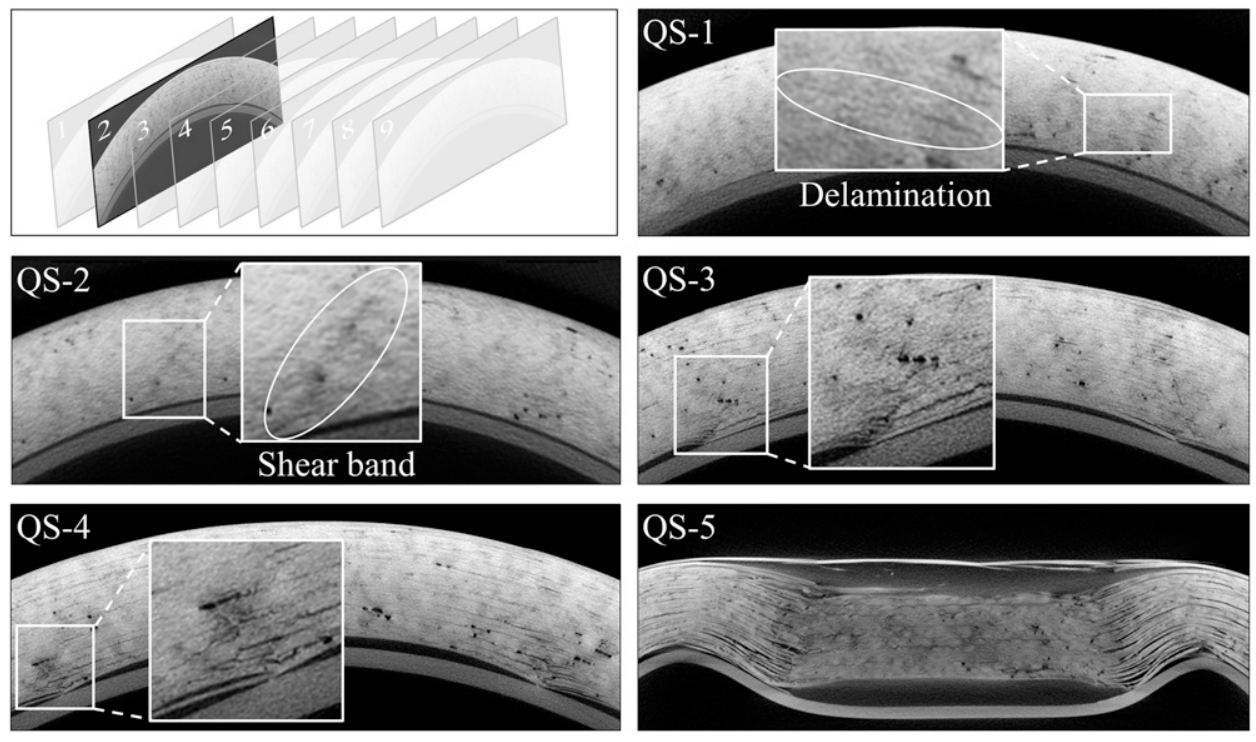

Figure 12. Computed tomography images of the quasi-statically loaded pressure vessels at location 2 (or 8 ) defined in Figure 10. 

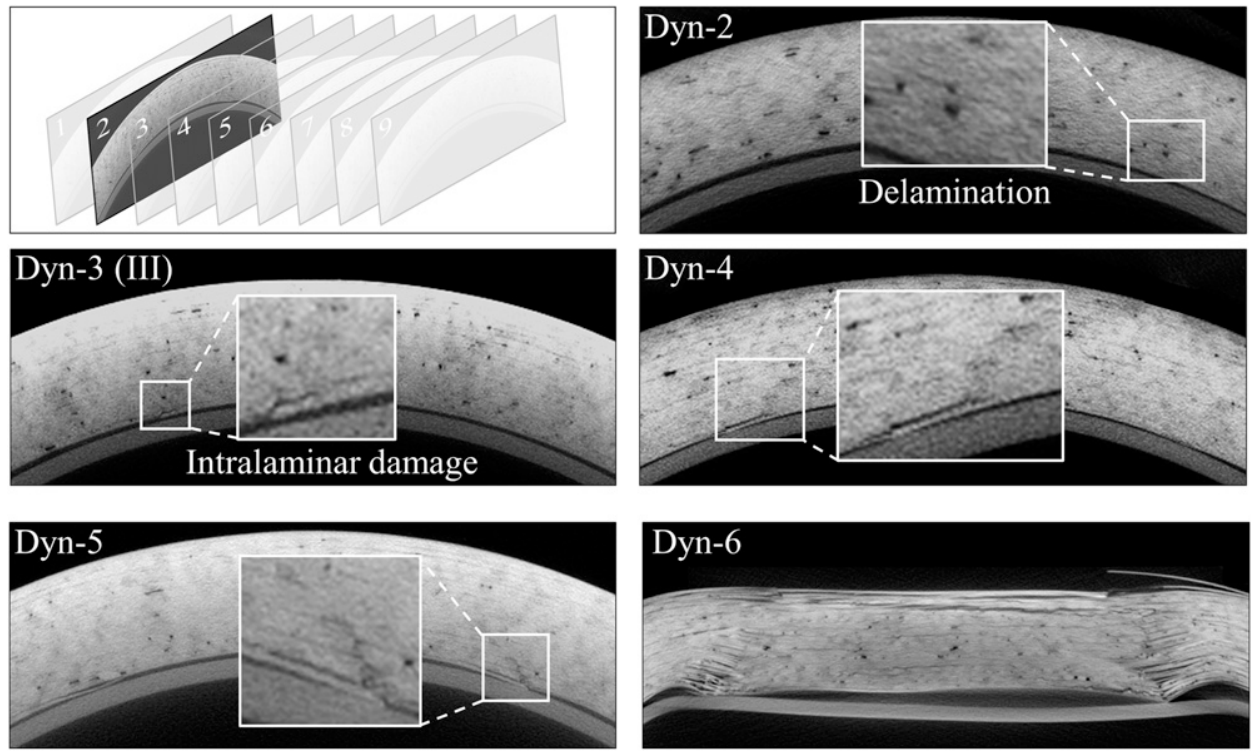

Figure 13. CT images of the dynamically loaded pressure vessels at location 2 (or 8) defined in Figure 10.

small delaminations may already be initiated before detecting them in the measured curves.

For increasing indenter displacement, additional delaminations develop and a shear band emerges as before, see QS-2. The initiation of the shear band is asymmetric and only visible on one side. It develops at the inner surface of the wall, hence away from the impactor. The shear band initiates at the left hand side in this case, but it may equally well initiate at the other side (for other samples). The image for QS-3 shows that the number of delaminations grows further and that the shear band becomes more distinct as the deformation increases. The initiation of a shear band at the other side can now also be identified. Note that no clear indication of shear band failure initiation and growth is present in the force-displacement curves, since no obvious irregularities are visible in the curves of samples QS-2 and QS-3 at displacements exceeding the maximum one of sample QS-1 (Figure 3). For sample QS-4, intralaminar damage that crosses entire plies occurs in both shear bands as some cracks develop in the radial direction. Moreover, the second shear band becomes more pronounced. Upon further loading, both observed shear bands grow across the entire thickness as seen for QS-5. The moments at which both shear bands extend across the entire wall thickness seem to correlate with the two force reductions at normalized displacements of approximately 0.5 and 0.8 , Figure 5. For the QS-5 pressure vessel, considerable permanent deformation is present.

For the dynamic case, the occurring damage is considerably more localized than for quasi-static loading, see Figure 13. Pressure vessel Dyn-1 is not included in the figure, as the visible damage only consisted of delaminations, as is the case for pressure vessel Dyn-2.
Pressure vessel Dyn-3(III) shows that intralaminar damage initiates at the innermost layers. The first failure mechanism extends for increasing energy levels, although remaining local, see Dyn-4. The shear band appears in pressure vessel Dyn-5, where a shear band develops at both sides, although being minor at the left hand side. The Dyn-6 case reveals that one of the shear bands (the right one in this case) extends across the wall thickness. This supports the earlier hypothesis that the force reductions in Figure 5 correspond to the shear band extension across the wall thickness. The normalized displacement of this pressure vessel is 0.62 , which exceeds the displacement at the first force drop. Based on this hypothesis, the second shear band extends across the thickness at a displacement of approximately 0.8 .

\section{Residual burst pressure}

The pressure vessels are pressurized to burst after the CT analysis using the procedure explained before. The residual burst pressures as a function of the normalized external work are presented in Figure 14. The burst pressures of four undamaged pressure vessels are included for reference.

Let us first focus on the quasi-statically loaded COPVs. The four asterisks from left to right correspond to QS-1 to QS-4. Sample QS-1 had a residual burst pressure that is within the typical range for undamaged pressure vessels. Hence, the imposed damage did not lead to a measurable reduction of the burst pressure. It can be concluded that delaminations as observed in Figure 12 do not affect the burst pressure. Note that the first reduction of burst pressure (QS-1 to QS-2) is significant and correlates with the nucleation of a shear band in the CT scan. The corresponding 


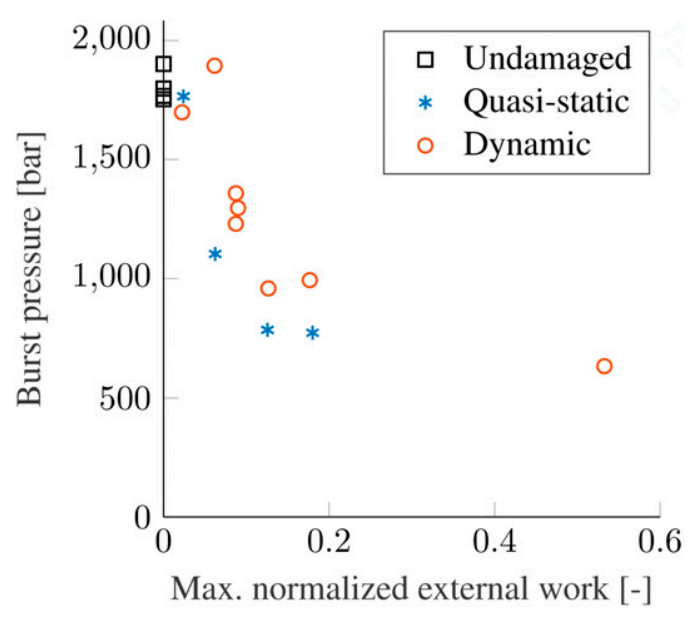

Figure 14. Residual burst pressure as a function of the maximum external work (normalized) for the tested samples.

reduction of burst pressure is thought to be due to local fiber fracture as a result of fiber kinking. A gradual progressive reduction of the residual burst pressure can be observed for samples QS-2, QS-3, and QS-4. The difference in residual burst pressure between samples QS-3 and QS-4 is small, even though the visual damage from Figure 12 suggests a considerable difference in damage severity.

The dynamic tests are indicated using circles. From left to right, the samples Dyn- 1 to Dyn-6 are shown, including the three repetitions Dyn-3(I) to Dyn-3(III). Samples Dyn-1 and Dyn-2 have a residual burst pressure that suggests no damage. This is in agreement with the earlier observation that only delaminations occur in this regime. Note that samples QS-2 and Dyn-2 are subjected to the same external work but that the residual burst pressures differ significantly. The residual burst pressure for the dynamic tests decreases sharply after exceeding a critical energy which is exceeded for the samples Dyn-3(I) to Dyn-3(III). The residual burst pressure of these samples subjected to the same incident energy is relatively consistent; the spread is large in the range of healthy pressure vessels, as evident from samples Dyn-1 to Dyn-2. This shows that the test procedure provides reproducible results. For increasing incident energies, the residual burst pressure does not drop as sharply anymore, an effect that also occurred in the quasi-static tests. Even for a large incident energy of 0.52 (sample Dyn-5), the residual burst pressure remains above 600 bar.

The comparison of the quasi-static and dynamic samples suggests that quasi-static loading causes more damage than dynamic loading for similar external work. Although the differences in burst pressure are generally within the typical spread for healthy pressure vessels, the overall analysis supports the following conclusion: The first reduction in burst pressure occurs at a larger energy in the dynamic case, and all samples that were tested at the same energy have a larger residual burst pressure in the dynamic case.
Moreover, the CT analyses also confirm that the observed damage mechanism (shear band formation) is less severe for dynamic loading.

\section{Conclusions}

A quasi-static and dynamic experimental study was performed to investigate the initiation and progression of damage in composite overwrapped pressure vessels subjected to contact loads. The behavior of pressure vessels under quasi-static loading conditions was highly reproducible, enabling to visualize the initiation and growth of damage. The first observed failure mechanism which leads to a considerable reduction in the force-displacement curve is assumed to be the initiation of delaminations. Although easily detectable in the measurement, the global residual strength of the COPV was not significantly affected by this failure mechanism. Upon further loading, shear bands and subsequent intralaminar failure emerged. These failure mechanisms were hardly visible in the force-displacement curve. The initiation of these shear bands correlated with a considerable reduction of the residual burst pressure, which is thought to be due to the formation of fiber fracture. These observations also apply to the pressure vessels loaded under dynamic conditions. The occurring failure was, however, much more localized, and shear band formation was only visible at larger deformations. Again, the initiation of intralaminar failure directly correlated with a reduction of burst pressure. In line with the more localized nature of damage under impact conditions, residual burst pressure tests also revealed that quasi-static loading is more severe than dynamic impact loading.

Future research should focus on the influence of the impactor geometry. In particular, the effect of smaller impactor geometries and imperfect contact (off-angle loading) should be investigated. Accordingly, the impact location is also a topic of open research. The investigated impacts were all in the cylindrical section of a pressure vessel; that is, impacts on the dome and boss were not considered. Moreover, the conducted tests considered pressure vessels without internal pressure. Preloading caused by internal pressure is expected to strongly affect the occurring failure mechanisms. To study the wide variety of open topics with reasonable raw material, time, and financial expenses, a numerical model is required. Such a model should be able to depict delaminations and shear band formation, and it should incorporate the three-dimensional nature of local loading.

\section{Declaration of conflicting interests}

The author(s) declared no potential conflicts of interest with respect to the research, authorship, and/or publication of this article.

\section{Funding}

The author(s) received no financial support for the research, authorship, and/or publication of this article. 


\section{ORCID iD}

Ruben AJ Weerts (D) https://orcid.org/0000-0002-7511-8623

\section{Note}

1. In the analysis of the data, the considerable reduction of velocity was defined as the frame in which the velocity was reduced by $2 \%$ with respect to the previous time frame.

\section{References}

1. Staffell I, Scamman D, Velazquez Abad A, et al. The role of hydrogen and fuel cells in the global energy system. Energy \& Environmental Science 2019; 12(2): 463-491. DOI: 10.1039/ C8EE01157E.

2. Díaz-González F, Sumper A, Gomis-Bellmunt O, et al. A review of energy storage technologies for wind power applications. Renewable and Sustainable Energy Reviews 2012; 16(4): 2154-2171. DOI: 10.1016/j.rser.2012.01.029.

3. Henning HM and Palzer A. What will the energy transformation cost? Pathways for transforming the German energy system by 2050. Germany: Fraunhofer ISE, 2015.

4. Sternberg A, Hank C and Hebling C. TreibhausgasEmissionen für Batterie- und Brennstoffzellenfahrzeuge mit Reichweiten über 300 km. Germany: Fraunhofer ISE 2019.

5. MacCharles A, Pocard N, Lin C, et al. Fueling the Future of Mobility: Hydrogen and Fuel Cell Solutions for Transportation. China: Deloitte-Ballard, 2020.

6. Robinius M, Linßen J, Grube T, et al. Comparative analysis of infrastructures: hydrogen fueling and electric charging of vehicles. Jülich, Germany: Schriften des Forschungszentrum Jülich Reihe Energie \& Umwelt / Energy \& Environment, 2018, 408.

7. Barthelemy H, Weber M and Barbier F. Hydrogen storage: Recent improvements and industrial perspectives. International Journal of Hydrogen Energy 2017; 42(11): 7254-7262. DOI: 10.1016/j.ijhydene.2016.03.178.

8. Moradi R and Groth KM. Hydrogen storage and delivery: Review of the state of the art technologies and risk and reliability analysis. International Journal of Hydrogen Energy 2019; 44(23): 12254-12269. DOI: 10.1016/j.ijhydene.2019. 03.041

9. Choi BH, Seo DC and Kwon IB. Detecting impact traces on a composite pressure vessel with aluminum-coating optical fiber using a phase-modulated BOCDA sensor. Composites Science and Technology 2017; 142: 264-274. DOI: 10.1016/j. compscitech.2017.02.019.

10. Saeter E, Lasn K, Nony F, et al. Embedded optical fibres for monitoring pressurization and impact of filament wound cylinders. Composite Structures 2019; 210: 608-617. DOI: 10.1016/j.compstruct.2018.11.051.

11. Liao B, Wang D, Hamdi M, et al. Acoustic emission-based damage characterization of $70 \mathrm{MPa}$ type IV hydrogen composite pressure vessels during hydraulic tests. International Journal of Hydrogen Energy 2019; 44(40): 22494-22506. DOI: 10.1016/j.ijhydene.2019.02.217.

12. Blanc-Vannet P, Bardoux O, Alexandre N, et al. Residual performance of composite pressure vessels submitted to mechanical impacts. In: International Conference on
Hydrogen Safety. Hamburg, Germany, 21 September 2021. DOI: $10.13140 /$ RG.2.2.20430.56646.

13. Blanc-Vannet P. Burst pressure reduction of various thermoset composite pressure vessels after impact on the cylindrical part. Composite Structures 2017; 160: 706-711. DOI: 10.1016/j. compstruct.2016.10.099.

14. Garcia M, Davis B and Miller J. Hypervelocity impact testing of a pressurized composite overwrapped pressure vessel and comparison to numerical analysis. Procedia Engineering 2017; 204: 476-483. DOI: 10.1016/j.proeng. 2017.09.744.

15. Demir I, Sayman O, Dogan A, et al. The effects of repeated transverse impact load on the burst pressure of composite pressure vessel. Composites Part B: Engineering 2015; 68: 121-125. DOI: 10.1016/j.compositesb.2014.08.038.

16. Perillo G, Grytten F, Sørbø S, et al. Numerical/experimental impact events on filament wound composite pressure vessel. Composites Part B: Engineering 2015; 69: 406-417. DOI: 10. 1016/j.compositesb.2014.10.030.

17. Weerts RAJ, Cousigné O, Kunze K, et al. A methodological approach to model composite overwrapped pressure vessels under impact conditions. Composite Structures 2020.

18. Giurgiutiu V. Vibration fundamentals. Structural Health Monitoring with Piezoelectric Wafer Active Sensors. Cambridge, MA: Academic Press, 2014, pp. 51-143.

19. Vogler TJ and Kyriakides S. On the initiation and growth of kink bands in fiber composites: Part I. Experiments. International Journal of Solids and Structures 2001; 38: 2639-2651.

20. Pimenta S, Gutkin R, Pinho S, et al. A micromechanical model for kink-band formation: Part I - Experimental study and numerical modelling. Composites Science and Technology 2009; 69(7-8): 948-955. DOI:10.1016/j.compscitech.2009. 02.010 .

21. Weerts RAJ, Cousigné O, Kunze K, et al. Assessment of contact-induced damage mechanisms in thick-walled composite cylinders. Journal of Reinforced Plastics and Composites 2020; 39(17-18): 679-699. DOI: 10.1177/ 0731684420923043.

\section{Appendix}

The force measurements in the dynamic contact tests are affected by a dynamic effect: the "dynamic amplification factor." For the adopted experimental setup, the existence and consequence of this effect can be shown by the use of a three mass-spring system. An illustration of the setup and the simplification of the components into masses and springs are shown in Figure 15. The mass $m_{i}$ resembles the mass of the indenter, the carriage, and any additional weights. An initial velocity matching the one of the physical test is prescribed to mass $m_{i}$. The support consisting of the support blocks, the base plate, and the load cells is modeled as mass $m_{s}$ and spring $k_{l}$, where the latter represents the stiffness of the load cells. The COPV is modeled as a mass $m_{C}$ with two springs $k_{C, u}$ and $k_{C, l}$. The two springs are aimed at modeling the deformation in the upper and lower half of the COPV, respectively. The stiffness of the two springs in 


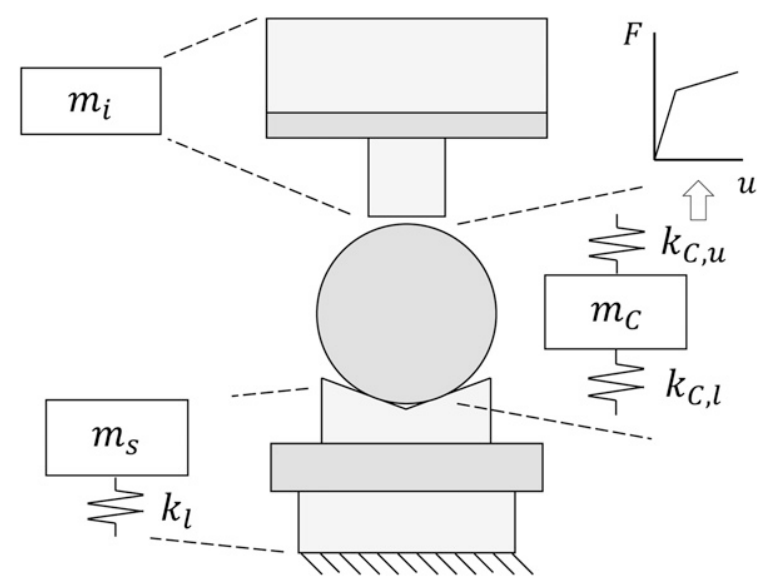

Figure I5. Simplified representation of the experimental setup as a mass-spring system. Failure in the COPV is incorporated through the nonlinear behavior of spring $k_{C, u}$.

series matches with that of the quasi-static measurement from Figure 3. Spring $k_{C, u}$ includes failure, which is represented using a bilinear curve that represents the quasi-static force-displacement curve in a simplified manner. Mass $m_{C}$ does not cover the entire weight of the COPV as the loading does not span the entire length, therefore causing a deformation mode that is not uniform along the pressure vessel. Hence, the weight was calibrated to obtain an approximate match with the results from Figure 15. The resulting weight is approximately half the physical mass.

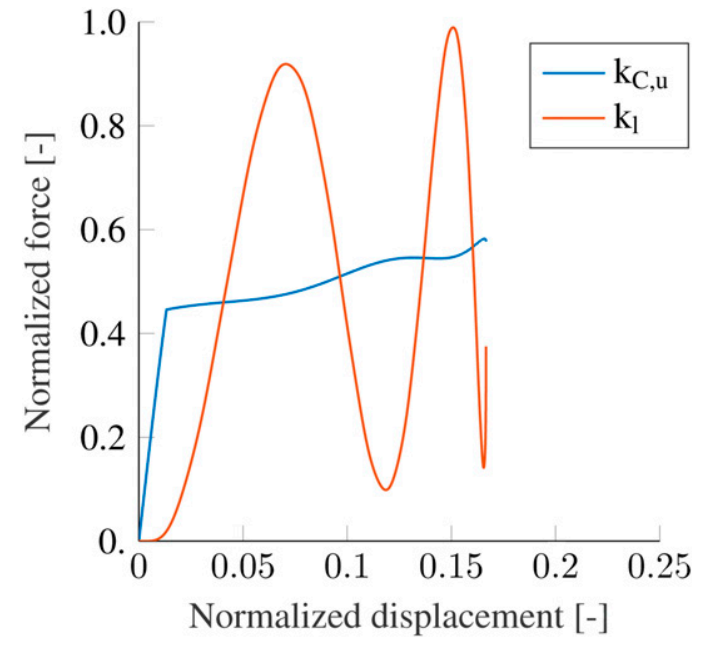

Figure 16. Normalized force-displacement curves for springs $k_{C, u}$ and $k_{l}$ from the mass-spring system in Figure 15.

The force-displacement curves in $k_{C, u}$ and $k_{l}$ are compared next, representing the failure and measurement location, respectively. Equivalent to the experimental study, the reported displacement is that of the impactor $\left(m_{i}\right)$. Figure 16 shows the resulting curves. The behavior is similar to that observed in Figure 7: The load cell measures the impact only after a delay and then oscillates around the curve for $k_{C}$, ${ }_{u}$, reaching approximately twice the force. Note that no damping is included in the model; therefore, the reduced amplitude observed in the experiment cannot be captured. 